\title{
Les enseignants polyvalents et l'activité de préparation des cours dans l'enseignement primaire : l'exemple de la géographie au cycle 3
}

\section{Philippe Charpentier et Daniel Niclot}

\section{(2) OpenEdition \\ Journals \\ Édition électronique \\ URL : http://journals.openedition.org/trema/2941 \\ DOI : 10.4000/trema.2941 \\ ISSN : 2107-0997 \\ Éditeur \\ Faculté d'Éducation de l'université de Montpellier}

\section{Édition imprimée}

Date de publication : 1 juin 2013

Pagination : 50 - 61

ISBN : 1167-315X

ISSN : 1167-315X

Référence électronique

Philippe Charpentier et Daniel Niclot, « Les enseignants polyvalents et l'activité de préparation des cours dans l'enseignement primaire : l'exemple de la géographie au cycle $3 »$, Tréma [En ligne],

39 | 2013, mis en ligne le 01 juin 2015, consulté le 19 avril 2019. URL : http://journals.openedition.org/ trema/2941 ; DOl : 10.4000/trema.2941

Ce document a été généré automatiquement le 19 avril 2019.

Trema 
Les enseignants polyvalents et l'activité de préparation des cours dans l'enseignement primaire : l'exemple de la géographie au cycle 3

Philippe Charpentier et Daniel Niclot

Introduction 
1 La connaissance du travail des enseignants $\mathrm{du}$ primaire et $\mathrm{du}$ secondaire fait partie des nouveaux objets de recherche investigués par des chercheurs appartenant aux IUFM. L'objectif des travaux menés sur le travail enseignant est généralement de mieux connaître les pratiques d'enseignement, le plus souvent dans un souci de formation professionnelle des enseignants. Les recherches qui sont menées s'appuient largement sur les champs scientifiques de l'analyse du travail (Leplat, 1997), la didactique professionnelle (Pastré, Mayen, Vergnaud, 2006) ou la clinique de l'activité (Clot, 2006) mais aussi sur les didactiques des disciplines.

Si beaucoup de recherches se sont centrées sur «ce que font" les maîtres durant les heures de classe, peu en revanche traitent

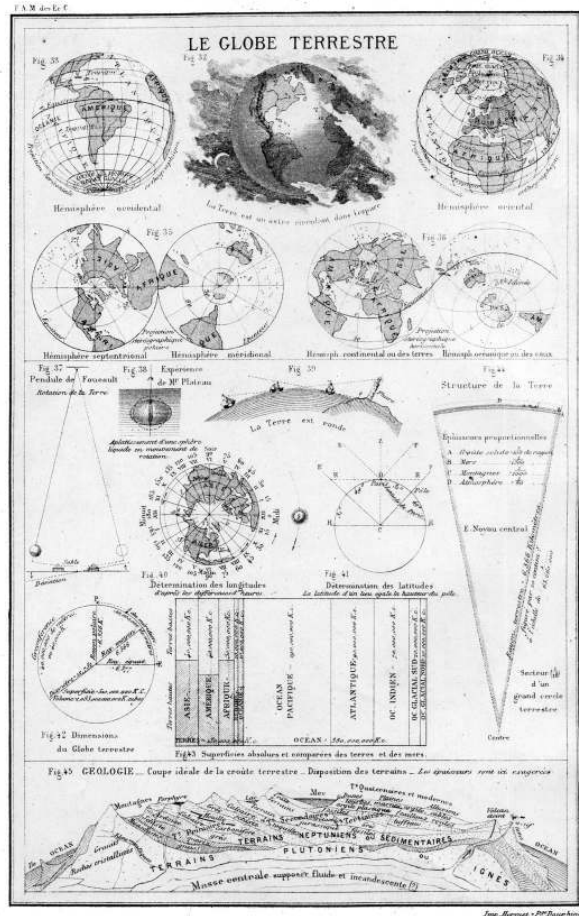
$\mathrm{du}$ travail enseignant hors $\mathrm{du}$ temps scolaire. C'est précisément l'objet de la recherche présentée ici qui vise à étudier le «temps caché » (Gueudet et Trouche, 2010) de la réalité de l'activité enseignante représentée par le temps de préparation des leçons, qui est à ce jour encore peu prise en compte par la recherche.

Le contexte général du travail des enseignants, aussi bien dans leur classe qu'hors de la classe, notamment lors de la préparation des leçons, est fortement conditionné par leur polyvalence qui peut être définie en première analyse comme une situation d'enseignement apprentissage caractérisée par « la prise en charge par chaque maître des différents domaines ou disciplines constitutifs du cursus » (Prairat et Rétornaz, 2002, p. 588). Alors que les maîtres du primaire, du fait même de leur polyvalence, ne sont spécialistes d'aucune discipline en particulier, une grande partie de leur activité, en cycle 3 notamment, est liée à l'enseignement de disciplines scolaires. Les enquêtes menées sur cette question ont montré (Baillat, Espinoza et Vincent, 2001) que si certains maîtres refusent d'entrer dans les logiques disciplinaires, ce qu'ils justifient par le fait que leur fonction est de favoriser l'épanouissement personnel des élèves, d'autres expriment leurs difficultés à maîtriser l'ensemble des disciplines et leur contenu.

4 À partir de l'exemple de l'enseignement de la géographie au cycle 3, cette recherche vise à aborder un sujet peu étudié, celui du travail de l'enseignant polyvalent confronté à la préparation des leçons de cette discipline et à en comprendre les logiques en mobilisant des concepts forgés dans le cadre de différents champs scientifiques relevant de l'analyse de l'activité. 


\section{Polyvalence et activité des maîtres de l'école primaire}

\section{1. Polyvalence et enseignement disciplinaire}

5 Les questions posées par la polyvalence, les pratiques des enseignants du primaire, notamment, les liens entre la polyvalence et les enseignements disciplinaires, ont fait l'objet de nombreux travaux en France depuis la fin des années 1990. Les recherches les plus nombreuses interrogent toutefois les pratiques d'enseignement disciplinaire des maîtres polyvalents (Baillat et Espinoza, 2009). En géographie, plusieurs enquêtes (Audigier 2000 ; Baillat et Niclot, 2000) mais aussi des études in situ sur les pratiques d'enseignement (Philippot, 2008) montrent que la géographie est une discipline qui est enseignée selon des horaires légèrement supérieurs à ceux préconisés par les instructions officielles. L'enseignement de cette discipline se caractérise par la prééminence des activités de localisation - nomination.

6 Si ces travaux permettent de mieux comprendre les caractéristiques du contexte de travail et les pratiques des enseignants du primaire, il apparaît toutefois qu'un moment important de l'activité enseignante, en l'occurrence la préparation des leçons, n'a fait l'objet d'aucune étude spécifique en langue française à l'exception de quelques communications dans des colloques de didactique des mathématiques (Leroyer et Bailleul, 2009 ; Margolinas et al, 2004).

\section{2. Cadre théorique : la préparation des leçons comme élément du réel de l'activité}

7 Le champ scientifique centré sur l'activité, issu de l'analyse du travail de langue française (Ombredane et Faverge, 1955), fournit un ensemble de concepts pertinents pour étudier la préparation des leçons par les maîtres du primaire. Dans le cadre de cette étude, trois courants sont mobilisés: l'ergonomie cognitive (Leplat, 1997), la didactique professionnelle (Pastré, Mayen et Vergnaud, 2006; Mayen, 2008) et la clinique de l'activité (Clot, 2004, 2008). Ils ont en commun de s'être d'abord intéressés au travail dans l'industrie et les services et de n'avoir abordé le travail enseignant que récemment.

\section{2. 1. L'ergonomie cognitive}

8 Pour Leplat (1997) l'activité ne se limite pas à la «production » proprement dite. Toute activité commence par une phase de préparation antérieure à l'action qui correspond à un temps de conception. Elle se prolonge éventuellement, après l'action, par des réflexions sur le travail effectué. Ces moments constituent ce que cet auteur appelle le réel de l'activité. L'ergonomie cognitive établit une autre distinction fondamentale en différenciant la tâche prescrite par l'institution et le travail réalisé qui n'épuise jamais totalement la prescription. Cet auteur considère que l'activité est dicible, qu'elle laisse des traces matérielles et non matérielles. En ce sens, elle peut être appréhendée par diverses méthodologies et notamment par des entretiens de nature variée. 


\section{2. 2. La didactique professionnelle}

9 La didactique professionnelle est un courant qui s'est développé au début des années 1990 en congruence avec le précédent. Son objet est d'analyser le travail en vue de la formation aux compétences professionnelles. Selon Pastré, Mayen et Vergnaud (2006) " elle cherche à articuler de façon très forte deux dimensions qui ne vont pas forcément ensemble : la dimension théorique et la dimension opératoire » (p. 151). La première permet d'éviter un discours empirique qui se contenterait de décrire, sans les expliquer, les opérations réussies. La seconde se rattache au courant de la conceptualisation dans l'action empruntée à Piaget et à Vygotski. L'hypothèse principale sur laquelle repose cette seconde dimension est que l'activité humaine est organisée sous forme de schèmes, dont le noyau central est constitué de concepts pragmatiques. Comme l'explique Mayen (2008), l'analyse de l'activité s'effectue dans le cadre de classes de situations, orientées vers un but, possédant une fonction spécifique et faisant partie d'un ensemble plus vaste, le réel de l'activité. Pastré, Mayen et Vergnaud (2006) insistent sur la variabilité des conduites et l'imprévisibilité des situations rencontrées par les professionnels dans une même classe de situations. Pourtant, à partir de plusieurs études, ils ont identifié des invariants opératoires, qui correspondent à des réponses semblables des professionnels face aux mêmes situations.

\section{2. 3. La clinique de l'activité}

L'objet de la clinique de l'activité est, pour Clot (2004), « moins de repérer la structure de l'activité en tant que telle que la structure de son développement possible ou impossible. L'objet de connaissance est moins l'activité que le développement de l'activité et ses empêchements. » (p.31). La dimension psychologique individuelle du travail est au cœur de la clinique de l'activité. Clot définit les attributs du concept d'activité en tenant compte du fait que l'activité est tout autant un objet social que psychologique. Reprenant Leplat, ce même auteur explique « que l'agent ne fait pas que réaliser la tâche prescrite, il vise aussi, par cette réalisation, des buts personnels » (Leplat, 1997, p. 28).

L'activité selon Clot (2000) est le siège d'investissements vitaux de la part de la personne, "le réel de l'activité est fait des activités suspendues, contrariées ou empêchées [...], contreactivités qui éventuellement l'empoisonnent» (p.133) explique cet auteur. "Vivre au travail c'est donc pouvoir y développer son activité, ses objets, ses instruments, ses destinataires, en affectant l'organisation du travail par son initiative " (Clot, 2008, p. 7). L'activité du sujet se trouve "désaffectée " lorsque cette initiative n'a plus prise sur l'activité. «On agit alors sans devenir actif [...] et cette désaffection diminue le sujet, le déréalise, non sans effet quant à l'efficacité de son action » (ibid.) et l'activité perd son sens.

12 Dans la logique de ces travaux, on peut considérer que la préparation de cours est un élément du réel de l'activité enseignante. La préparation des leçons constitue une classe de situations qui correspond à une phase de conception et comme telle, elle « ne se réduit pas à un ou deux objets, avec leurs propriétés et relations; on y rencontre des acteurs, des enjeux, des obstacles, des moyens, des outils, et une temporalité » (Vinatier, 2009, p. 28). En ce sens, notre hypothèse est que par des enquêtes et des entretiens menés sur la préparation des leçons par les maîtres du primaire il est possible d'analyser leur activité dans le cadre de cette classe de situations, de dévoiler une partie du réel de l'activité 
" cachée ", de mettre en évidence des variabilités et des invariants opératoires mais aussi les structures cognitives de la tâche qu'ils construisent et des tensions; notamment liées à des écarts entre la tâche prescrite et l'activité effective.

\section{Enquête sur la préparation des leçons de géographie}

\section{1. Méthodologie}

13 L'activité de préparation des leçons correspond à un temps "caché » selon l'expression employée par Gueudet et Trouche (2008). Elle s'effectue en dehors des heures de classes, le plus souvent au domicile de l'enseignant et à des moments variés. Aussi en raison des difficultés que rencontrerait le chercheur pour observer in situ cette activité, le recueil de données a été réalisé sous forme de questionnaires et d'entretiens menés auprès des maîtres de cycle 3 de l'enseignement primaire.

Une première enquête par questionnaire a été effectuée par contact interpersonnel direct, sans passer par des canaux hiérarchiques, auprès d'enseignants de cent sept écoles du Val-de-Marne de février à mai 2010. Le questionnaire comporte quatre parties: l'identité du maittre, les difficultés liées à la préparation des leçons, les supports utilisés et la formation initiale ou continue. 113 réponses ont été obtenues de la part de 21 hommes et 91 femmes (une personne interrogée n'a pas répondu à cette question). Les enseignants exercent selon des proportions sensiblement égales dans les trois niveaux du cycle 3, CE2, CM1 et CM2 et 11 ont des classes à double niveaux. 69 \% des répondants ont entre 26 à 40 ans. Les trois quart ont un niveau universitaire supérieur ou égal à trois années d'étude après le Baccalauréat.

Comme le questionnaire comporte des questions fermées, il a semblé intéressant de mener, dans un second temps, des entretiens semi-directifs qui ont eu lieu au dernier trimestre de l'année 2011. 14 maîtres qui enseignent effectivement la géographie en cycle 3 ont été interrogés dont trois quarts avaient déjà répondu à la première enquête.

Les données obtenues sont interprétées en mobilisant les concepts du champ scientifique de l'analyse de l'activité afin de mieux comprendre la nature, les caractéristiques et le sens du travail des enseignants polyvalents du primaire lorsqu'ils préparent des cours disciplinaires, en l'occurrence, la géographie. Cette recherche se situe donc dans une perspective méthodologique spécifique, celle qui consiste à inviter des enseignants à verbaliser ce qu'ils font. Comme le note Bressoux (2000), les données recueillies correspondent à « un discours sur la pratique, non la pratique elle-même; or, il faut se garder d'assimiler pratiques déclarées et pratiques effectives. [...]. Les discours mettent en œuvre des processus de reconstruction et de rationalisation: le répondant cherche généralement à rendre son discours cohérent, or la pratique ne revêt pas nécessairement une telle cohérence » (p. 132). Les écarts ou les distorsions entre le dire et le faire peuvent être liés au fait que les acteurs n'ont pas la mémoire de tous leurs faits et gestes, ou n'ont pas les outils conceptuels pour mettre en mots leur pratique (Lahire, 2005). 


\section{2. La préparation, une classe de situation spécifique, un élément du réel de l'activité}

On peut considérer, en reprenant un des concepts du champ de la didactique professionnelle, que la préparation des leçons correspond à une classe de situations, c'està-dire un temps «diachronique » de l'activité (Tochon, 1989) orienté vers un but qui comprend la conception et l'organisation de la leçon et qui se poursuit par un temps « synchronique » d'enseignement auprès des élèves.

Si l'on s'attache à la durée de préparation des leçons de géographie, il apparait que cette classe de situations représente une part importante du réel de l'activité. En effet, 82 enseignants sur les 110 ayant répondu à la question, soit les trois quarts, déclarent passer de 10 et 40 minutes par heure d'enseignement pour préparer une leçon de géographie. On peut noter cependant que trois maîtres déclarent passer moins de dix minutes et dix plus d'une heure. Il n'y a pas de différence significative selon le niveau de classe concerné (CE ou CM).

19 Au total, pour une leçon de géographie d'une heure en cycle 3, on peut estimer, si l'on s'en tient aux déclarations, que le réel de l'activité correspond généralement à $1 \mathrm{~h} 30$ ou 1 h40 de travail auquel il faut ajouter le temps consacré aux corrections. Ce temps de préparation relativement important peut être mis en relation avec la réponse à une autre question dans laquelle plus de $90 \%$ des enseignants estiment que l'enseignement de la géographie est très important ou important pour l'éducation des élèves.

\section{3. Les finalités de l'enseignement de la géographie et la tâche prescrite}

20 À la question portant sur les finalités de l'enseignement de la géographie à l'école primaire, les enseignants devaient choisir parmi plusieurs propositions celles qui leur semblaient les plus importantes. Trois quarts des enseignants interrogés répondent que l'enseignement de la géographie aide les élèves à comprendre le monde qui les entoure. Trois autres propositions qui attribuent respectivement à cette discipline une dimension culturelle, civique et de "préservation de la terre» totalisent plus de la moitié des réponses.

21 Les représentations qu'ont les maîtres sur les finalités de la géographie sont donc diverses et oscillent entre une perspective de formation intellectuelle de l'individu qu'est l'élève et des finalités collectives, tournées vers l'utilité sociale. Dans les entretiens menés en complément du questionnaire, une autre finalité apparait de manière plus ou moins explicite. Une jeune enseignante explique : « et puis, en règle générale, la géographie, les élèves, ils aiment bien aussi parce que cela sort un peu des disciplines basiques: le Français... ». Cette remarque confirme l'idée que les maîtres du primaire attribuent à la géographie une fonction " récréative ", soulignée par ailleurs dans la plupart des travaux qui ont analysé les pratiques d'enseignement de la géographie dans l'enseignement primaire (par exemple Audigier, 1999 ; Philippot, 2008).

22 La pluralité des finalités citées correspond en fait à celle qui figure dans les différents programmes. Ceci n'est guère surprenant car un nombre important d'enseignants déclare connaître les textes officiels régissant l'enseignement de cette discipline. 72 enseignants 
sur 113 affirment s'appuyer sur les programmes pour élaborer leur progression et 77 déclarent lire les textes officiels lors des changements de programme en géographie, mais aussi dans les autres matières. On peut donc globalement estimer qu'une majorité de maîtres possède une connaissance assez détaillée de la tâche prescrite.

\section{4. La structure cognitive de la tâche}

La connaissance de la tâche à effectuer ne résout pas pour autant les problèmes de préparation. Plus de la moitié des maîtres interrogés par questionnaire, soit 63 personnes, estiment avoir des difficultés à préparer les leçons de géographie. Toutefois, 49 enseignants déclarent ne pas éprouver de difficultés particulières dont $20 \%$ parmi ces derniers avouent un manque d'intérêt pour la discipline.

Les trois obstacles principaux rencontrés par les maitres pour préparer les leçons de géographie sont la difficulté à trouver des documents pertinents puis le manque de temps et l'absence de connaissances précises sur la discipline. Au-delà de ces obstacles, l'insuffisance de formation en géographie est souvent considérée comme la source principale des difficultés éprouvées. Elle concerne aussi bien la formation professionnelle initiale que continue puisqu'un enseignant sur dix seulement a suivi un stage en géographie.

Quelles que soient les difficultés éprouvées par les maîtres, des conceptions majoritaires sur les méthodes d'apprentissage de la géographie orientant leur activité de préparation apparaissent. Parmi les propositions du questionnaire, deux sont privilégiées : «faire localiser sur la carte » (36\%) et « c'est par l'analyse de document que l'élève construit ses connaissances géographiques » (44\%). Les autres propositions obtiennent, quant à elles, des résultats inférieurs à $10 \%$. À la question "à quoi sert la documentation recherchée ", les enseignants interrogés répondent presqu'à l'unanimité (103 sur 113 réponses), «à illustrer la leçon » et à « introduire la leçon » (82 sur 113 réponses).

On retrouve dans ces résultats une double dimension soulignée par les chercheurs qui ont travaillé sur les pratiques d'enseignement de la géographie (Baillat et Niclot, 2000; Audigier 1999; Philippot 2008 ; Baillat et Espinoza, 2009). Il s'agit d'abord de l'importance accordée par les maîtres du primaire à la mise en activité des élèves qui se traduit par l'utilisation de documents illustratifs et introductifs et ensuite au rôle attribué à la localisation.

L'écart est important entre les prescriptions, connues de la plupart des maîtres qui citent les différentes finalités intellectuelles, culturelles et sociales de l'enseignement de la géographie et les pratiques d'enseignement majoritaires qui privilégient des exercices de localisation et la mise en activité des élèves. La compréhension de ces écarts peut être référée au concept de structure cognitive de la tâche qui «s'interpose " entre les prescriptions et l'activité réelle. Cet écart peut s'expliquer par le fait qu'en raison de leur insuffisante maîtrise des contenus et des enjeux éducatifs de la discipline, beaucoup d'enseignants adaptent leur activité aux dimensions spécifiques et objectives de la situation qu'ils perçoivent, à leur conception des apprentissages et au manque de temps dont ils disposent pour préparer leurs leçons. 


\section{5. Invariants opératoires}

28 L'une des questions centrales du questionnaire concerne les sources de la documentation utilisée par les maîtres. Un premier résultat significatif est que tous les enseignants interrogés sans exception déclarent utiliser les manuels scolaires comme source de documentation dans leur activité de préparation. Le ou les manuels sont d'autant plus appréciés qu'ils proposent un « livre du maître ».

Un autre invariant opératoire est constitué par l'orientation de l'activité de préparation des leçons de géographie vers la recherche de documents. Parmi les documents recherchés en géographie, deux types dominent ; cités par près des 100 enseignants sur les 113 interrogés, les photographies (parmi lesquelles les images de paysages) et les cartes. Comme dans la plupart des classes les élèves ne disposent pas d'un manuel de géographie, les enseignants conçoivent des dossiers documentaires. La procédure décrite dans quelques entretiens est la suivante : après avoir sélectionné les documents, ceux-ci sont photocopiés (parfois en couleur) puis distribués aux élèves pour servir de support à leur mise en activité. Comme l'explique un enseignant, le choix du manuel sur lequel s'appuie le maître est lié en grande partie à la qualité des photocopies qu'il permet de faire.

Le recours systématique aux manuels scolaires constitue un invariant opératoire commun à l'ensemble des enseignants, qui, au-delà de l'enseignement de la géographie semble d'ailleurs concerner toutes les disciplines comme l'indiquent les déclarations faites lors des entretiens.

\section{6. Variabilité des pratiques}

31 Au-delà de ces invariants, les pratiques de préparation se caractérisent par une grande variabilité, notamment en ce qui concerne l'utilisation de l'Internet. Il faut noter à ce propos que son usage est en partie lié à l'âge puisque les enseignants âgés de moins de quarante ans sont proportionnellement plus nombreux à l'utiliser que les plus âgés (six sur dix pour quatre sur dix).

Globalement, un peu moins de $60 \%$ des enseignants déclarent consulter des sites dans le cadre de la préparation de leurs cours. L'encyclopédie collaborative Wikipedia, les sites institutionnels et privés sont utilisés par plus d'un maître sur deux. "Je commence souvent par Wikipedia parce que tu as des textes pas mal, tu as des images de qualité et en haute résolution » déclare une jeune enseignante. Une autre utilise l'Internet, faute de trouver les documents qu'elle recherche dans les manuels : « ce que je fais, c'est que je me rabats sur les sources que je peux trouver sur internet ».

Plusieurs enseignants utilisent les sites institutionnels ou privés à destination des maîtres du primaire, mais parfois avec beaucoup de précaution :

« Mais vraiment, je fais très attention, quand par exemple, c'est des choses qui sont mises en ligne par des instits, qui ne sont pas validées. Je lis, j'en prends connaissance, mais je ne prends pas pour argent comptant parce que je sais très bien qu'ils n'ont pas le recul, ni les qualifications. On n'est pas tous agrégés de géographie ».

Dans une société où l'usage de l'Internet se généralise, permettant à chacun d'accéder à une multitude de documents et d'informations, l'usage de cet outil peut apparaître 
comme une solution «nouvelle» au problème de la recherche de documents. Pourtant son usage ne s'est pas généralisé à l'ensemble de la profession. En tous cas, il n'a pas détrôné les manuels scolaires, sources traditionnelles de documentation des enseignants comme le signalaient déjà les résultats d'une enquête publiées en 1988 par Tournier et Navarro.

Enfin, quand les maîtres ont un doute sur un point du programme, ils prennent conseil auprès de connaissances ou de leurs collègues (plus de six enseignants sur dix). «J'ai la chance d'avoir ma meilleure amie qui a une licence de géographie. Donc elle me valide ou elle m'invalide ce que je pense être... » confie une enseignante lors d'un entretien.

\section{7 Désaffection et tensions}

L'activité de préparation de leçons disciplinaires par les maîtres polyvalents est source de tensions voire de désaffections qui s'expriment dans plusieurs entretiens. Deux sources de tensions sont généralement citées qui rendent difficile la mise en œuvre des prescriptions officielles : le manque de temps nécessaire à la préparation des leçons de géographie et l'insuffisance de formation des enseignants polyvalents dans la discipline. Le plus souvent les préparations se font «à la maison» comme le soulignent plusieurs enseignants, ce qui nécessite d'effectuer des arbitrages entre le temps à consacrer à la préparation des leçons des différentes disciplines, dont certaines sont jugées «plus fondamentales » que d'autres et sa vie personnelle ou familiale.

Un enseignant de CM1 qui possède une expérience de huit années d'enseignement avoue avoir utilisé un seul manuel pour préparer ses leçons de géographie durant ses premières années d'enseignement. Il semble le déplorer implicitement et il se sent obligé de justifier cette situation par une nécessité vitale, celle de la préservation de son temps de sommeil :

"La première année, tu galères. Parce que l'inspecteur m'avait dit : ciblez deux matières. On ne peut pas tout faire. Ciblez deux matières, il m'avait dit : maths ou français à fond. Et puis, vous êtes bon en sciences, faites-vous plaisir en sciences. Tu fais maths et français. Il arrive un moment où il faut corriger, il faut dormir. Et oui, j'avais trouvé cela (le manuel de géographie) et... je l'avais vraiment utilisé clé en main. "

Une enseignante chevronnée évoque également la question du temps de préparation qui est de toute évidence pour elle une source de tension voire d'épuisement professionnel :

«...mes collègues viennent souvent me demander des trucs parce qu'elles savent ... que je m'intéresse à mon travail. Je suis assez comme ça. Donc, je n'ai pas de limites... Donc, moi, oui, cela va durer encore trois, quatre ans. Je pense qu'après je prendrais ma retraite. Voilà. Je ferai autre chose. Je ne suis pas malheureuse de partir, au contraire."

Cependant grâce à l'expérience, une gestion rationnelle du temps peut amoindrir la tension et être un « soulagement » comme l'explique un autre enseignant expérimenté :

"Non. Je ne refais pas (mes cours) tous les ans mais, eh bien, encore une fois, j'évolue. Je deviens peut-être un petit peu plus efficace ».

Le manque de formation professionnelle initiale et continue en géographie et son corollaire, la nécessité de se former seul, pose avec plus d'acuité encore la question du temps. Elle est à l'origine de formes de « désaffection », pour reprendre le concept de Clot (2008), ce qui signifie agir sans devenir actif en nuisant à l'efficacité de l'action.

39 À la question du chercheur sur la formation qu'elle a reçue en géographie une jeune enseignante (deux années d'expérience) explique : 
"C'était super rapide en fait. C'était plus sur l'organisation générale d'une séance... je me suis vachement appuyée sur les livres. Je suis ce qu'il y a (dans les manuels), parce que sinon... ". qu'elle n'a pas eu de véritable formation en géographie :

"On te donne des billes pour t'en sortir en classe. On consacre beaucoup sur le français et les mathématiques, à juste titre, compte tenu des programmes. Mais ce qui concerne les matières dites culturelles, humanistes. On est livré à soi-même."

41 Les remarques d'un professeur des écoles qui enseigne depuis plus de trente ans font écho à celles des plus jeunes, même si elles sont plus nuancées :

«Pour la géographie comme pour les autres matières, j'estime que je me suis formé sur le tas aux dépens des premières générations d'élèves que j'ai eues. Et, je dois dire, qu'encore maintenant, je réalise que je ne suis pas au point sur tout. J'ai encore à changer des choses et je découvre encore de nouvelles pistes de travail ».

Hors la question du temps insuffisant pour préparer ses leçons et de l'absence de formation, quelques autres sources de tension sont mentionnées dans les entretiens. Il s'agit par exemple des difficultés éprouvées pour s'adapter aux évolutions ou au niveau supposé des élèves. Une enseignante de plus de cinquante ans évoque des élèves de plus en plus difficiles à intéresser. Une autre insiste sur la nécessité de développer des activités simples et que l'essentiel est « d'aider les enfants à comprendre. Parce qu'ils ne sont pas d'une complexité débordante. » Ces assertions restent toutefois exceptionnelles.

\section{Conclusion}

43 Le « dire sur le faire » exprimé par les réponses au questionnaire et les déclarations faites lors des entretiens mettent en évidence les difficultés éprouvées par les maîtres polyvalents dans leur activité de préparation des leçons de géographie. Pour reprendre une expression employée par une enseignante lors d'un entretien, ils ont le sentiment d'être « livrés à eux-mêmes » ce qui constitue une source de tension voire de désaffection. Ils manquent de connaissances didactiques, de repères et d'outils. Ils en sont conscients et ils le disent. L'explication de cette situation se trouve, d'après les déclarations des maitres, autant dans les carences de leur formation professionnelle que dans le manque de temps qu'ils peuvent consacrer à la préparation des diverses disciplines à enseigner.

Ce constat rejoint les résultats de la recherche effectuée par Philippot (2008) sur les pratiques d'enseignement et les discours des enseignants centrée sur l'enseignement de la géographie. L'insuffisance de maîtrise des disciplines scolaires se traduit par des pratiques d'enseignement qui, bien souvent, s'en tiennent aux aspects les plus formels, aux tâches les plus « rituelles ». Les enseignants orientent leur activité de préparation en fonction de la structure cognitive de la tâche qu'ils ont construite en adaptant leur préparation, et en amont leurs leçons, à la nécessité de mettre en activité les élèves et de favoriser les exercices de localisation. Dans ces conditions, l'activité de préparation n'est pas tournée vers une réflexion sur la mise en œuvre des programmes pour faire acquérir aux élèves des savoirs, des savoir faire ou des compétences mais elle s'oriente vers la recherche de documentation.

Si ces constats concernent le cas de la géographie, en se référant aux exemples donnés lors des entretiens, on peut émettre l'hypothèse que les pratiques concernant la préparation des leçons sont assez semblables dans la plupart des autres disciplines. 
Nouvelle méthode de géographie, Cours supérieur. Atlas universel (première partie) : le ciel et la terre, p.9, s.d.. Editeurs Bricon, J. et Lesot, A., Paris. Source : CEDRHE : 5086.

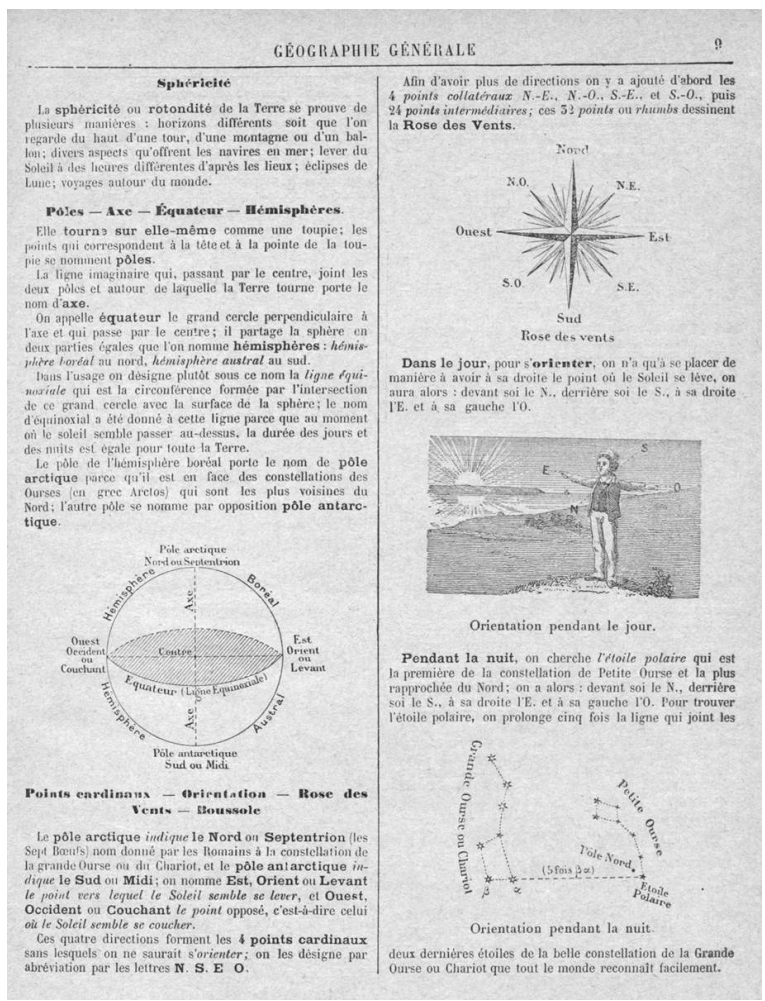

\section{BIBLIOGRAPHIE}

Audigier, F., Les représentations de la géographie dans l'enseignement primaire en France, habitat commun, voisinage et distance. Cahiers de géographie du Québec, n¹20, n43, 1999, p. 395-412.

Baillat, G. et Niclot, D., L'histoire et la géographie à l'école primaire. Une discipline scolaire à part entière ou deux matières d'enseignement ? ». In G. Baillat (dir) Enquête sur la polyvalence des maîtres du premier degré. Rapport de recherche, Reims : IUFM Champagne Ardenne, 2000, p. 81-85.

Baillat, G. et Espinoza, O. (dir.)., Les enseignants du primaire et les savoirs scolaires, 2009.

Bisault, J., Objectivations et convergences dans les moments scolaires de sciences au cycle 3. In Symposium "éducation scientifique à l'école primaire et professionnalité des enseignants », Bisault, J. (Resp.), Communication présentée au Congrès international AREF 2010 - Actualité de la Recherche en Éducation et en Formation. Genève -septembre 2010.

Bisault, J. et Berzin, C., Analyse didactique de l'activité effective des élèves en sciences à l'école primaire. Éducation et didactique, n³, n², 2009, p. 81-103. 
Bressoux, P., Modélisation et évaluation des pratiques et des environnements d'enseignement. Grenoble : Université Pierre Mendès France, 2000.

Clot, Y., La formation par l'analyse du travail : pour une troisième voie. In Y. Clot Manières de penser, manières d'agir en éducation et en formation. Paris : PUF, 2000, p. 133-156.

Clot, Y., Action et connaissance en clinique de l'activité. Activités, nº1, 2004, p. 23-33.

Clot, Y. (2008). Travail et pouvoir d'agir. Paris : PUF, 2008.

Gueudet, G. et Trouche, L., Des ressources aux documents, travail du professeur et genèses documentaires. In G. Gueudet et L. Trouche (dir.) Ressources vives, Rennes : PUR, 2010, p. 21-37.

Lahire, B., L'esprit sociologique. Paris : La Découverte, 2005.

Leplat, J. (1997). Regards sur l'activité. Paris : PUF.

Leroyer, L. et Bailleul, M., Les enseignants travaillent aussi hors la classe : comment? Actes du colloque International Espace Mathématique Francophone. Enseignement des mathématiques et développement. Enjeux de société et de formation. 2009, avril. http://fastef.ucad.sn/EMF2009/Groupes \%20de\%20travail/GT2\%20et\%209/Groupe\%20N\%B02.html

Margolinas, C., Canivenc, B., De Redon, M.C., Rivière, O. et Wozniak, F., Que nous apprend pour la formation des maîtres le travail mathématiques hors classe des professeurs? Dans Actes du XXXIe colloque Copilerem des professeurs et des formateurs de mathématiques chargés de la formation des maîtres. Quelles mathématiques faire vivre à l'école? Quels outils pour les maitres ? IREM Toulouse, 2004, mai. http://arpeme.fr/documents/5250C9775C52373ABCAD.pdf

Mayen, P., Intégrer les savoirs à l'action. In Perrenoud, P., Altet, M., Lessard, C. et Paquay, L. (dir.), Conflits de savoirs en formation des enseignants. Entre savoirs issus de la recherche et savoirs issus de l'expérience. Bruxelles : De Boeck, 2008, p. 43-58.

Ombredane, A. et Faverge, J.M., L'analyse du travail : facteur d'économie humaine et de productivité. Paris : PUF, 1955.

Pastré, P. Mayen, P. et Vergnaud, G., La didactique professionnelle. Revue française de pédagogie, $\mathrm{n}^{\circ}$ 154, 2006, p. 144-198.

Philippot, T., La professionnalité des enseignants de l'école primaire : les savoirs et les pratiques. Thèse de doctorat, Université Reims Champagne-Ardenne, Reims, 2008.

Sachot, M. et Lenoir, Y. (dir.), Les enseignants du primaire entre disciplinarité et interdisciplinarité : quelle formation didactique? Québec : Presses de l'Université Laval, 2004.

Tournier, M et Navarro, M., Les professeurs et le manuel scolaire, Rapport de recherche $\mathrm{n}^{\circ} 5$. Paris : INRP, 1988.

Tochon, F., À quoi pensent les enseignants quand ils planifient leurs cours ? Revue Française de Pédagogie, Paris, n86, 1989, p. 23-33.

Vinatier, I. et Altet, M. (dir.), Analyser et comprendre la pratique enseignante. Rennes : Presses universitaires de Rennes, 2008.

\section{RÉSUMÉS}

À partir d'un questionnaire et d'entretiens, la recherche présentée est centrée sur la préparation des leçons de géographie par des maîtres enseignant en cycle trois.

Les résultats mettent en évidence de forts écarts entre les prescriptions et l'activité effective, 
l'existence d'invariants opératoires malgré la diversité des pratiques de préparation des leçons et une orientation vers la mise en activité des élèves. L'activité de préparation de leçons est source de tensions et des formes de désaffection s'observent qui s'expliquent, selon les maîtres, par leur manque de formation professionnelle dans cette discipline et de temps disponible.

From a questionnaire and from interviews, the research is centered on the preparation of the lessons of geography by teachers teaching in cycle three of the french primary school.

The results highlight strong distances between the prescriptions and the effective activity, the existence of operating invariants in spite of the diversity of the practices of preparation of the lessons and an orientation towards the activation of the pupils. The activity of preparation of lessons is source of tensions and forms of disaffection are observed by the teachers which are understandable according to them by their lack of vocational training in this discipline and of available time for the preparations.

INDEX

Keywords : activity, geography, preparation of the lessons, primary education, teacher of french primary school

Mots-clés : activité, enseignement primaire, géographie, polyvalence, préparation des leçons

\section{AUTEURS}

PHILIPPE CHARPENTIER

CEREP (EA 4692)

DANIEL NICLOT

CEREP (EA 4692) 